\title{
Blink Frequency and Duration during Perimetry and Their Relationship to Test-Retest Threshold Variability
}

\author{
Yanfang Wang, ${ }^{1}$ Sonia S. Toor, ${ }^{2}$ Ramesh Gautam, ${ }^{1}$ and David B. Henson ${ }^{1}$
}

Purpose. To describe different patterns of blinking in patients undergoing a visual field test and to establish whether the blink parameters are related to threshold variability.

MeTHoDs. Thirty-nine patients with diagnosed or suspected glaucoma were recruited to undertake a perimetric task twice. Blinks were detected with a video eye-tracker system that records at a sampling rate of $60 \mathrm{~Hz}$. Blink frequency, duration, and episodes of microsleep (eye closures $>500 \mathrm{~ms}$ ) were analyzed, and correlated with test-retest threshold variability. The timing of blinks with respect to stimulus presentation was analyzed and the percentage of seen stimuli for all presentations (POS ${ }^{\text {overall }}$ ) and those overlapped with blinks (POS ${ }^{\text {over }}$ lapped) were compared.

REsults. Blink frequency ranged from 0 to 58 per minute. A significant increase in blink frequency was observed in the second test $(P<0.001)$, whereas blink duration and microsleep episodes were not significantly different between the two tests. The relationship between test-retest threshold variability and all blink parameters was not significant. For suprathreshold stimulus presentations, blinks often occurred after presentation, whereas for subthreshold presentations, their timing was independent of stimulus timing. The difference between POS ${ }^{\text {overall }}$ and POS ${ }^{\text {overlapped }}$ was significant $(P<$ 0.001), and a slight decrease in POS ${ }^{\text {overlapped }}$ was observed with the increase of overlap duration.

Conclusions. A wide range of blink frequencies was observed during perimetric testing. Although no blink parameters showed significant influence on threshold variability, when the blinks overlapped with a stimulus presentation, the probability of seeing was reduced. For suprathreshold stimuli, blinks often occurred after the presentation, whereas for subthreshold presentations, there was no relationship to presentation time. (Invest Ophthalmol Vis Sci. 2011;52:4546-4550) DOI: 10.1167/iovs.10-6553

B links are not uncommon during a perimetric test, and it is well recognized by perimetrists that blink frequency varies from one patient to another. Although some patients hardly ever blink during a visual field test, others show frequent blinking that often appears to be related to stimulus presenta-

From the ${ }^{1}$ School of Biomedicine, Manchester University, Manchester, United Kingdom; and the ${ }^{2}$ School of Psychology, University of Reading, Reading, United Kingdom.

Supported by the Manchester Academic Health Sciences Centre (MAHSC) and the NIHR (National Institute of Health Research) Manchester Biomedical Research Centre.

Submitted for publication September 10, 2010; revised January 12 and February 7, 2011; accepted March 21, 2011.

Disclosure: Y. Wang, None; S.S. Toor, None; R. Gautam, None; D.B. Henson, None

Corresponding author: David B. Henson, Manchester University, Manchester Royal Eye Hospital, Oxford Road, Manchester, M13 9WH, UK; david.henson@manchester.ac.uk. tion times. At present, there is no literature on the frequency of blinks during perimetry or whether there is a relationship between blink parameters (frequency and duration) and perimetric findings such as threshold variability.

Blink frequency and duration have been associated with the performance of other attentional tasks (e.g., driving, piloting aircraft $)^{1-4}$ and correlate with subjective sleepiness scales ${ }^{5}$ and the EEG. ${ }^{6-8}$ Suppression or reduction of blinking occurs with tasks that have a high visual attention demand, such as reading. ${ }^{9}$ Blink duration correlates with increased sleepiness or fatigue $^{10-12}$ and may provide a more significant index for alertness than blink frequency. ${ }^{13}$

Loss of attention has been related to variability in the visual field $^{14}$ and, given the above relationships for other vigilance tasks, blink frequency and duration could provide an objective measure of attention during a perimetric test.

The purposes of present study were fourfold:

1. To report on the frequency and duration of blinks in a sample of perimetrically experienced patients attending a glaucoma outpatient clinic.

2. To report on the timing of blinks with respect to stimulus presentation.

3. To establish whether the frequency and duration of blinks are related to threshold variability.

4. To establish the effect of blinking during a stimulus presentation.

\section{Methods}

\section{Subjects}

Thirty-nine patients (age, $63.2 \pm 14.0$ years; range, $20-85 ; 17$ women and $22 \mathrm{men}$ ) with diagnosed or suspected glaucoma and prior experience with perimetry were recruited from the outpatient clinic of Manchester Royal Eye Hospital. Patients with advanced visual field loss (MD $<-12 \mathrm{~dB}$ ) or medication that may affect pupil size (e.g., pilocarpine) were excluded. The study was approved by the local research ethics committee and adhered to the tenets of the Declaration of Helsinki. Consent was obtained from each patient for participation.

\section{Apparatus}

The experimental equipments consisted of a visual stimulus generator (VSG, version 1.03; Cambridge Research System [CRS], Cambridge, UK), display monitor, eye-tracker $(250 \mathrm{~Hz}$ High Speed Video Eye Tracker [HS-VET]; CRS), response box, and host computer. Custom software (Delphi, ver. 7; Embarcadero, San Francisco, CA) was used both to measure the central visual field (full-threshold 24-2 automated perimetry, $0.5^{\circ}$ stimulus, $200-\mathrm{ms}$ presentation time, and $10-\mathrm{cd} / \mathrm{m}^{2}$ background luminance) at a testing distance of $330 \mathrm{~mm}$ and to record eye blink parameters. Eye blink parameters were recorded with an infrared-sensitive camera at a sampling rate of $60 \mathrm{~Hz}$, which provided an objective, noninvasive, precise, and continuous measure of eye tracking in real time. 


\section{Experimental Tasks}

The data were collected in a quiet, dimly illuminated room. The patients were required to perform the 24-2 full-threshold perimetric test twice with the same eye (the other eye was occluded). The interstimulus interval was adjusted automatically according to the patient's response times, starting off with an interval of $2000 \mathrm{~ms}$ and varying between 1600 and $2850 \mathrm{~ms}$. Fixation loss was tested with presentations at the blind spot and false-positive and -negative responses were tested with catch trials. A break of approximately 2 minutes was given between the two tests. This testing protocol is similar to that used in the routine management of glaucoma, where both eyes are tested.

\section{Data Analysis}

Eye Blink Detection. Eye blinks were defined as eyelid closures with a duration of 50 to $500 \mathrm{~ms} .^{10,13}$ Closures in excess of $500 \mathrm{~ms}$ were defined as microsleep episodes. ${ }^{13}$ The timing of each blink was also measured with respect to stimulus onset times and separately for stimuli above and below each test locations' measured threshold.

Visual Field Test. Indices extracted from the visual field data were threshold variability and percentage of seen stimuli (POS).

Threshold Variability. Threshold variability is defined as the standard deviation of the test-retest threshold sensitivity differences at all tested locations (excluding the blind spot) for each patient.

POS. The POS was obtained for all stimulus presentations (POS ${ }^{\text {overall }}$ ) and separately for presentations when a blink partially or fully overlapped with a presentation (POS $\left.{ }^{\text {overlapped }}\right)$. POS ${ }^{\text {overlapped }}$ was calculated for various overlap durations ( $>0, \geq 50, \geq 100$, and $\geq 150 \mathrm{~ms}$ ).

Statistical Analysis. Blink parameters (blink frequency, mean duration, and number of microsleeps) were calculated for both tests of each patient. Intertest differences of blink duration and threshold sensitivity were compared by paired $t$-test, whereas those of blink frequency and microsleep numbers were analyzed with the Wilcoxon signed ranks test. For patients with different blink patterns, intergroup difference in blink frequency and duration were also compared. Correlation analysis was used to look for relationships between threshold variability and blink parameters. Comparison of threshold variability between patients with random or temporally stimulus-related blinks was analyzed with independent-samples $t$-tests. The difference between POS ${ }^{\text {overall }}$ and POS ${ }^{\text {overlapped }}$ was also analyzed (all analyses: SPSS 16.0; SPSS, Chicago, IL). The significance level was 0.05 .

\section{Results}

\section{Blink Characteristics}

The mean (SD) frequency of blinks for the first and second tests was 9.96 (10.46) per minute and 13.90 (12.16) per minute. Most of the patients $(30 / 39 ; 76.9 \%)$ showed an increase in blink frequency in the second test, and the difference was significant $(Z=-3.503 ; P<0.001)$. Figure 1 gives the frequency of blinks for each patient in the first and second tests, ordered according to frequency in the first test.

Figure 1 also gives the mean blink duration for each patient and test (excluding microsleeps). Blink duration was normally distributed, with a mean of $153.5 \pm 46.7 \mathrm{~ms}$ in the first test and $163.1 \pm 42.3 \mathrm{~ms}$ in the second $\left(t_{38}=-1.665 ; P=0.104\right)$. There was no obvious relationship between blink frequency and blink duration (first test: $r=0.034, P=0.836$; second test: $r=0.039, P=0.812$ ).

The number of microsleep episodes in the first and second tests are also shown in Figure 1. Of the patients, 33.3\% had
Figure 1. Blink frequency (top), blink duration (middle), and number of microsleep episodes (bottom) for each patient in the first (gray columns) and second (black columns) tests.
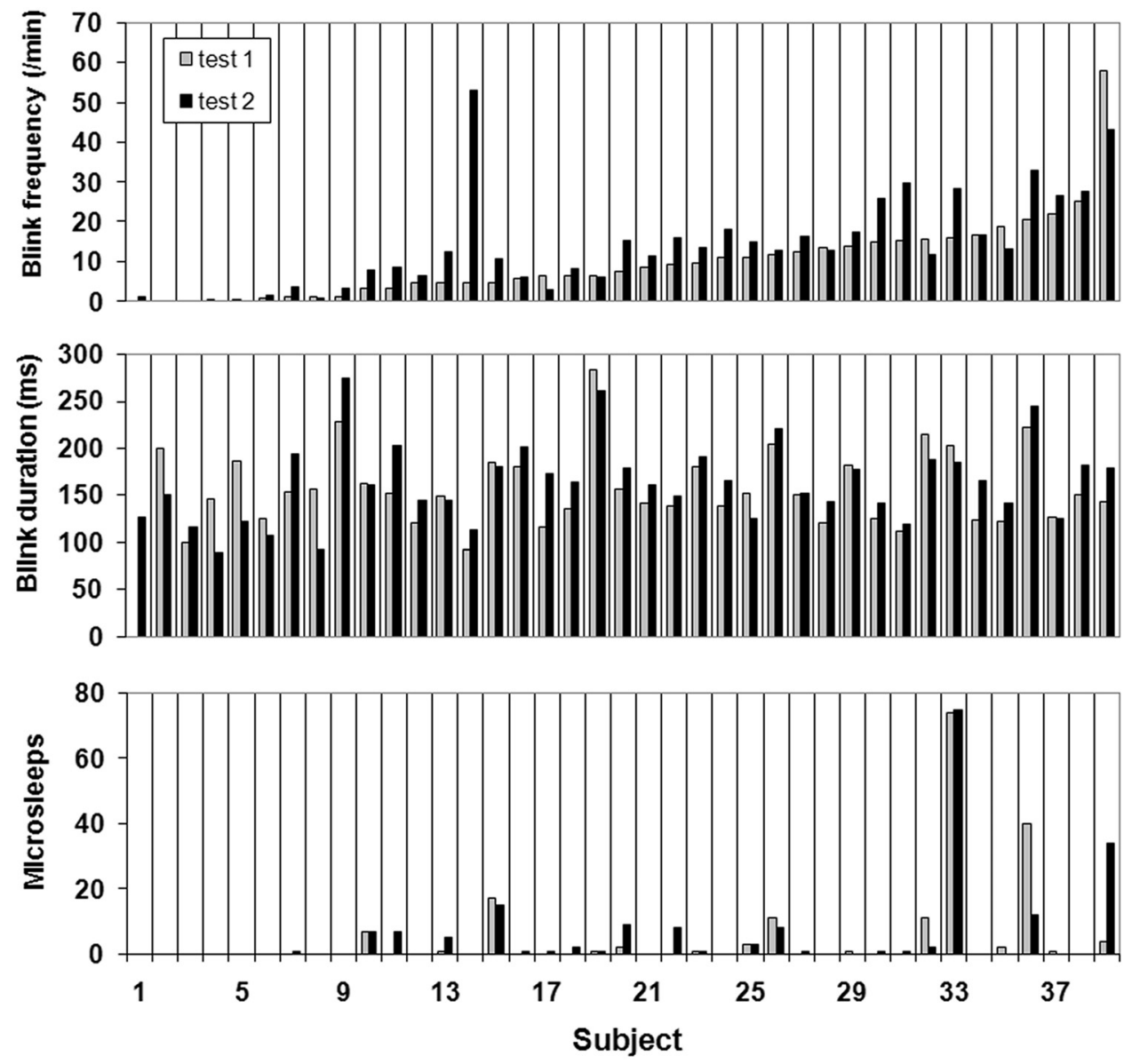

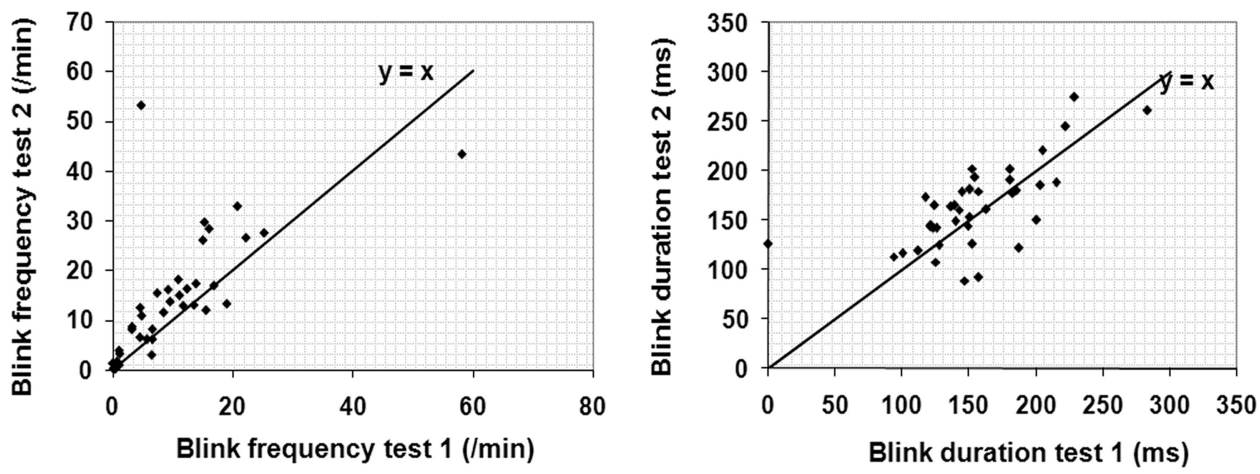

Figure 2. Blink frequency and duration in the first and second tests. Slope: equality of the displayed parameters. more microsleeps in the second test, whereas $17.9 \%$ had less and $35.9 \%$ had no microsleeps in either test. The difference in number of microsleeps was not significant in the two tests $(Z=-0.868 ; P=0.385)$.

There was good agreement between both frequency (mean [SD] difference: test 1 minus test $2,-3.94$ blinks/min [9.00]) and duration of blinks (mean difference: test 1 minus test 2 , $-9.56 \mathrm{~ms}$ [35.85]) in the first and second tests, with one notable exception, patient 14 , who had a dramatic increase in blink frequency during the second test. The consistency of blink frequency and duration in the first and second tests is illustrated in Figure 2.

\section{Blinks and Stimulus Presentation}

Cumulative plots of eyelid closure during a blink, time locked to stimulus onset $(-500$ to $+1500 \mathrm{~ms}$ ), were generated for all tests.

Sixteen tests had very few blinks $(<2 / \mathrm{min})$, and thus it was impossible to establish any valid relationship with stimulus presentation.

In the 62 tests with blink frequencies more than two per minute, 34 of 62 showed an increase in eyelid closures after stimulus presentation (see example in Fig. 3). In 16 of 62 tests, eyelid closures were observed to peak both before and after stimulus presentations and in 12 of 62 tests, there were no obvious peaks of eyelid closure (Fig. 3). There was no significant difference in blink frequency and duration between groups of different blink patterns (blink frequency: $\chi^{2}=2.817$, $P=0.244$; blink duration: $F_{2,61}=1.812, P=0.172$ ).
When the timing of blinks was plotted separately for stimuli above and below the measured threshold, the increased frequency after stimulus presentation was seen to be largely confined to situations in which the stimulus was suprathreshold (Fig. 4).

\section{Correlation between Blink Parameters and Threshold Variability}

A significant difference of mean threshold sensitivity was observed between the first and second tests (first test: $15.38 \pm$ $3.28 \mathrm{~dB}$; second test: $\left.14.73 \pm 3.89 \mathrm{~dB} ; t_{38}=3.79, P=0.001\right)$. The mean test-retest threshold variability was $4.10 \pm 1.58 \mathrm{~dB}$. The relationship between blink parameters and threshold variability is illustrated in Figure 5, which shows no significant correlation between threshold variability and averaged blink frequency $(r=0.019, P=0.910)$, blink duration $(r=0.118$, $P=0.473)$, and number of microsleeps $(r=0.150, P=0.364)$. For subjects with different blink patterns related to stimulus presentation, no significant difference in threshold variability was observed between subjects who blinked randomly and those who showed a temporal relationship to stimulus presentation $(t=1.042, P=0.306)$.

\section{Blinks during Stimulus Presentation}

For the tests when subjects had blinks greater than two per minute, the difference between POS ${ }^{\text {overall }}$ and POS ${ }^{\text {overlapped }}$ was significant $(P<0.001)$. When the overlaps were $>0$ $\mathrm{ms}$, most of the patients (47/62 tests) showed a smaller

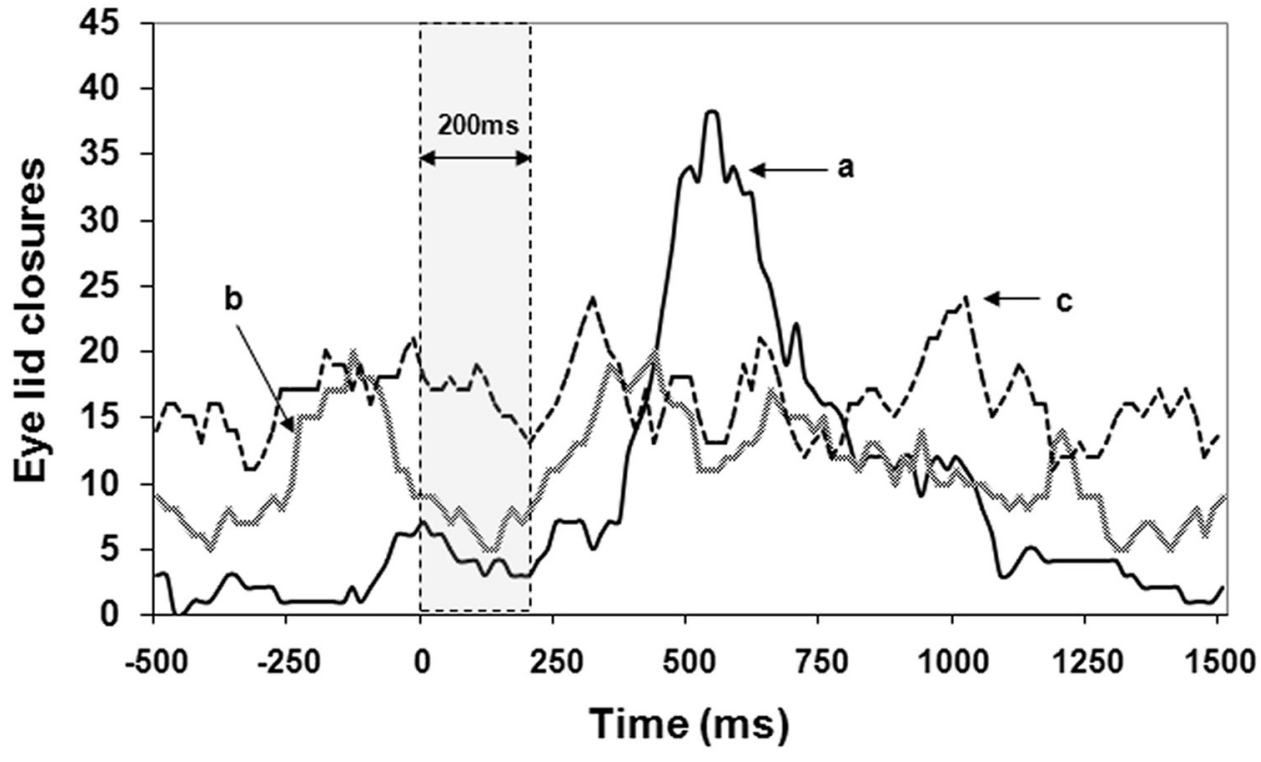

Figure 3. Cumulative plots of blink eyelid closures, time-locked to stimulus presentation, in three patients: (a) one who showed an increased frequency after stimulus presentation; (b) another who showed an increased frequency both before and after stimulus presentation; and (c) another who showed no relationship to stimulus presentation. Shaded ar$e a$ : stimulus presentation time. 
Group 1

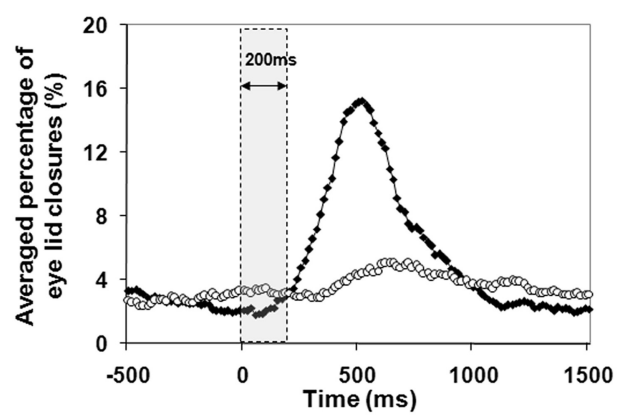

Group 2

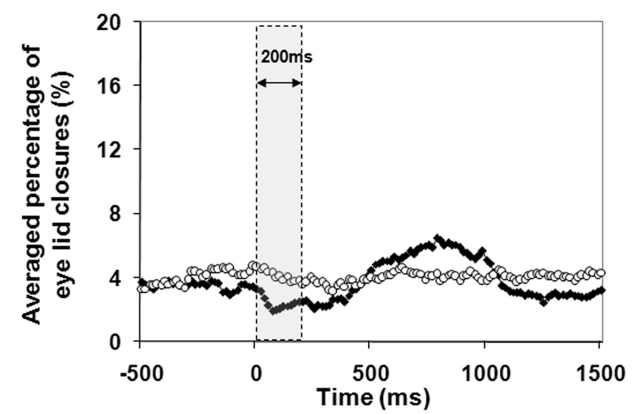

Figure 4. Average percentage of eyelid closures before and after stimulus presentations within each of the three groups (group 1, increased frequency after stimulus presentation, $n=34$; group 2 , increased frequency before and after stimulus presentation, $n=16$; group 3 , no pattern related to stimulus presentation, $n=12$ ). Data plotted separately for conditions where the presenting stimulus was above and below each test location's measured threshold. Percentage of eyelid closures was obtained by dividing the number of closures under defined stimulus conditions by the number of presentations (above or below threshold).

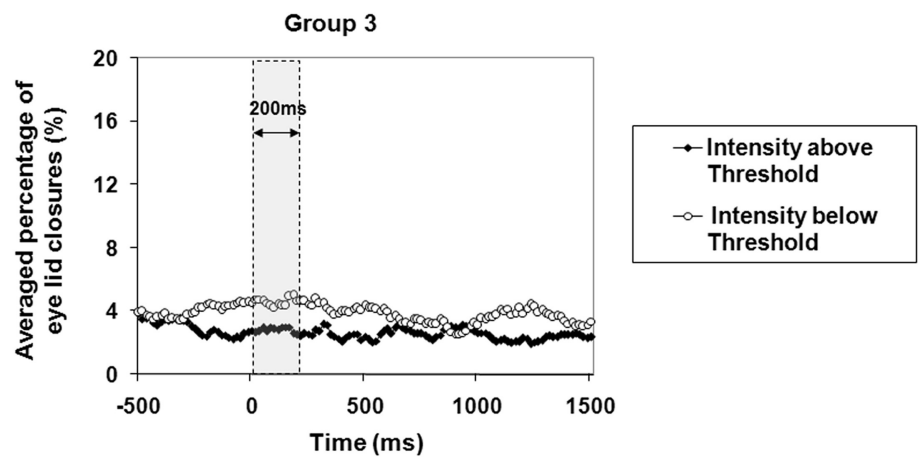

POS ${ }^{\text {overlapped }}$ than POS ${ }^{\text {overall }}$ (overall: $44.5 \% \pm 5.5 \%$; overlapped: $\left.31.9 \% \pm 22.1 \% ; t_{61}=4.796, P<0.001\right)$. When the overlap duration was $\geq 50, \geq 100$, and $\geq 150 \mathrm{~ms}$, POS ${ }^{\text {overlapped }}$ was $31.7 \%$ ( $\pm 23.8 \%), 28.3 \%$ ( $\pm 26.3 \%)$, and $26.8 \%( \pm 33.1 \%)$, which showed a slight decrease in POS with increasing overlap (Fig. 6). For those presentations that overlapped with blinks at the onset and offset point, POS was 29.3\% ( $\pm 24.8 \%$ ) and $33.6 \%$ ( $\pm 27.1 \%$ ), respectively (Fig. 6).
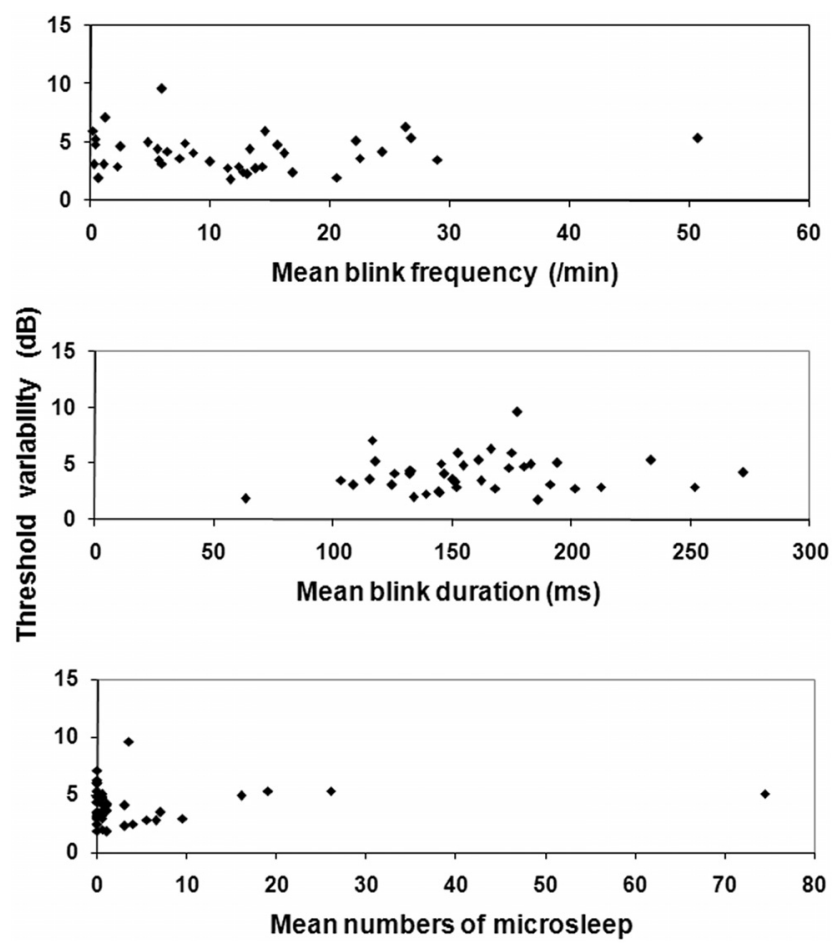

FIGURE 5. Relationship between blink parameters and threshold variability.

\section{Discussion}

The present study is the first to report on the frequency and characteristics of blinks in patients undergoing automated perimetry. Some patients were found to suppress blinking for long periods during the perimetric test, whereas others had a very high frequency of blinks (range, $0-58.09 / \mathrm{min}$ ). Normal blink frequency is on the order of 9 to 13 per minute in the daytime, ${ }^{5,15}$ increasing to 20 to 30 per minute in sleep-deprived subjects or patients with abnormal sleep patterns. ${ }^{6,16}$ On repeat testing, most of the patients showed a small increase in the number of blinks. Although this is most likely the results of an increase in fatigue during the second test, we cannot rule out the possibility that it is a learning effect.

Blink duration also varied between patients but again showed a high degree of intrapatient consistency varying little, either within a test or between the two tests. Caffier et al. ${ }^{10}$ reported significant changes in blink duration during the day

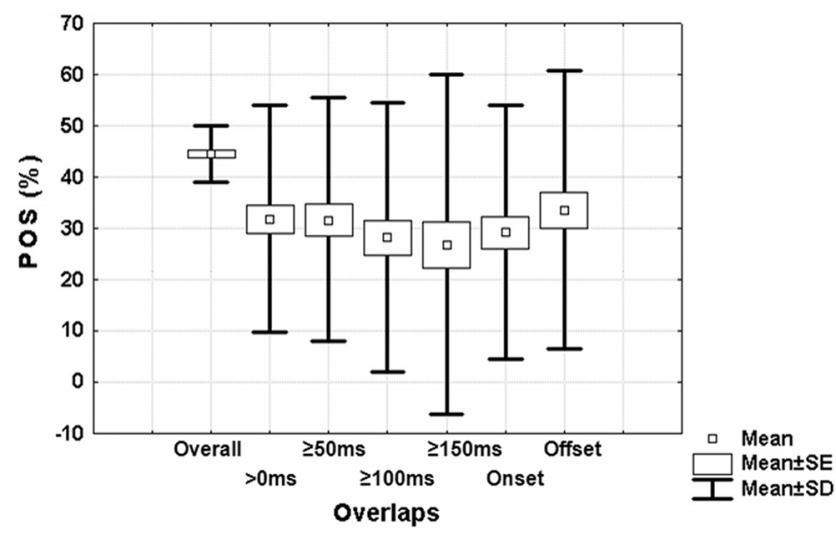

FIGURE 6. Overall POS and POS with various extents of overlap between blinks and stimulus presentations $(>0, \geq 50, \geq 100$, and $\geq 150$ ms onset and offset points) for 62 tests with a blink frequency of more than two per minute. 
that are related to sleepiness. During the relatively short period of the visual field test $(<10$ minutes), no such changes were observed, despite there being evidence that attention varies during a visual field test. ${ }^{17}$ Microsleep episodes, which are considered to be an intermediate stage between drowsiness and stage 1 of sleep, ${ }^{13,18}$ were rare in the present study, which may also be accounted for by the relatively short duration of our perimetric tests in comparison to that of tests in other studies of blinks.

When we looked at the temporal relationship of blinks to stimulus presentation, it was clear that there were three different patterns in our sample: (1) blink frequency peaking after a presentation; (2) blink frequency peaking both before and after a presentation, and (3) blinks unrelated to stimulus presentation. However, when the data were separately analyzed for stimulus presentations that were above and below threshold, it was clear that seeing the stimulus had an effect on the blink pattern of those falling in groups 1 and 2. Blinking after stimulus presentation was largely confined to situations where the patient saw the stimulus. When it was not seen (below threshold) blinks were no longer related to the stimulus presentation. Similar blink patterns have been reported in studies of auditory or visual stimuli in discriminative tasks. ${ }^{19,20}$ In these studies blinks occurred most frequently between 300 and $1000 \mathrm{~ms}$ after stimulus onset which is similar to that reported here. Several papers have reported that blinks are suppressed during periods of high attentive loading. ${ }^{9,21}$ The suppression of blinks during stimulus presentation reflects both a high state of attention and an ability to predict when the next stimulus is likely to occur.

Correlation analysis shows no relationship between blink parameters (blink frequency, blink duration, and microsleep) and test-retest threshold variability. In subjects with different stimulus-related blink patterns, there is also no significant difference of threshold variability observed. This study included patients with early to moderate visual field loss (MD $>-12$ ) and there is a strong relationship between variability and threshold sensitivity ${ }^{22-24}$ that may have masked any small effect due to blink parameters.

It was not surprising that the percentage of seen stimuli decreased when the blinks occurred during stimulus presentation, and there was a small reduction in the percentage of seeing with increasing overlap. Percentage of seeing is a simple but relatively crude measure of performance that is likely to be affected by the extent of any visual field loss and the patient's response characteristics (false positive rate). The reduction in the percentage of seen stimuli although small signifies a change in performance that is likely to increase threshold variability. Currently, patients are not given specific instructions with regard to blinking during a perimetric test, and further work is needed to see whether specific instructions would alter blink patterns and whether this would have any meaningful effect on overall variability. An option on many perimeters is to have an auditory stimulus (a bleep) linked to the stimulus presentation. Auditory stimuli act as a cue to stimulus presentation and may assist patients in predicting when the next stimulus is about to be presented. Several perimetrist prefer not to use auditory cues, as they believe this encourages false-positive responses. Further work is needed to establish the benefit or otherwise of providing an auditory stimulus.

In conclusion, a wide range of blink frequencies and patterns of blinking occur during routine perimetry. Most patients show an increased frequency of blinks after suprathreshold presentations, and the likelihood of seeing a stimulus decreases when a blink coincides with the presentation of a stimulus.

\section{References}

1. Bergasa LM, Nuevo J, Sotelo MA, Barea R, Lopez ME. Real-time system for monitoring driver vigilance. IEEE T Intell Transp. 2006; 7(1):63-77.

2. Stern JA, Boyer D, Schroeder DJ. Blink Rate as a Measure of Fatigue: A Review. Washington DC: FAA Civil Aeromedical Institute; 1994;1-12.

3. Morris TL, Miller JC. Electrooculographic and performance indices of fatigue during simulated flight. Biol Psychol. 1996;42:343-360.

4. Brookings JB, Wilson GF, Swain CR. Psychophysiological responses to changes in workload during simulated air traffic control. Biol Psychol. 1996;42:361-377.

5. Barbato G, Ficca G, Muscettola G, Fichele M, Beatrice M, Rinaldi F. Diurnal variation in spontaneous eye-blink rate. Psychiatry Res. 2000;93(2):145-151.

6. Barbato G, Ficca G, Beatrice M, Casiello M, Muscettola G, Rinaldi F. Effects of sleep deprivation on spontaneous eye blink rate and alpha EEG power. Biol Psychiatry. 1995;38(5):340-341.

7. Varner JL, Rohrbaugh JW, Sirevaag EJ, Packingham K, Stern JA. Time-frequency signal analysis applied to EEG signals associated with eye blinks. Annu Int Conf IEEE Eng Med Biol Proc. 1996;3: 1013-1014.

8. Bonfiglio L, Sello S, Andre P, et al. Blink-related delta oscillations in the resting-state EEG: A wavelet analysis. Neurosci Lett. 2009; 449(1):57-60.

9. Bentivoglio AR, Bressman SB, Cassetta E et al. Analysis of blink rate patterns in normal subjects. Mov Disord. 1997;12(6):1028-1034.

10. Caffier PP, Erdmann U, Ullsperger P. Experimental evaluation of eye-blink parameters as a drowsiness measure. Eur J Appl Physiol. 2003;89(3):319-325.

11. Ingre M, ÅKerstedt T, Peters B, Anund A, Kecklund G. Subjective sleepiness, simulated driving performance and blink duration: examining individual differences. J Sleep Res. 2006;15(1):47-53.

12. Sugiyama K, Nakano T, Yamamoto $S$ et al. Method of detecing drowsiness level by utilizing blinking duration. JSAE Review. 1996; 17:159-163.

13. Schleicher R, Galley N, Briest S, Galley L. Blinks and saccades as indicators of fatigue in sleepiness warnings: looking tired? Ergonomics. 2008;51(7):982-1010.

14. Miranda MA, Henson DB. Perimetric sensitivity and response variability in glaucoma with single-stimulus automated perimetry and multiple-stimulus perimetry with verbal feedback. Acta Opbthalmol. 2008;86(2):202-206.

15. De Padova V, Barbato G, Conte F, Ficca G. Diurnal variation of spontaneous eye blink rate in the elderly and its relationships with sleepiness and arousal. Neurosci Lett. 2009;463(1):40-43.

16. Caffier PP, Erdmann U, Ullsperger P. The spontaneous eye-blink as sleepiness indicator in patients with obstructive sleep apnoea syndrome-a pilot study. Sleep Med. 2005;6(2):155-162.

17. Henson DB, Emuh T. Monitoring vigilance during perimetry with pupillography. Invest Opbthalmol Vis Sci. 2010;51(7):3540-3543.

18. Blaivas AJ, Patel R, Hom D, Antigua K, Ashtyani H. Quantifying microsleep to help assess subjective sleepiness. Sleep Med. 2007; 8(2):156-159.

19. Fukuda K. Analysis of eyeblink activity during discriminative tasks. Percept Mot Skills. 1994;79:1599-1608.

20. Fukuda K. Eye blinks: new indices for the detection of deception. Int J Psychophysiol. 2001;40(3):239-245.

21. Nakano T, Yamamoto Y, Kitajo K, Takahashi T, Kitazawa S. Synchronization of spontaneous eyeblinks while viewing video stories. Proc $R$ Soc B: Biol Sci. 2009;276:3635-3644.

22. Chauhan BC, Tompkins JD, LeBlanc RP, McCormick TA. Characteristics of frequency-of-seeing curves in normal subjects, patients with suspected glaucoma, and patients with glaucoma. Invest Ophthalmol Vis Sci. 1993;34:3534-3540.

23. Heijl A, Lindgren A, Lindgren G. Test-retest variability in glaucomatous visual fields. Am J Ophthalmol. 1989;108(2):130-135.

24. Henson DB, Chaudry S, Artes PH, Faragher EB, Ansons A. Response variability in the visual field: comparison of optic neuritis, glaucoma, ocular hypertension, and normal eyes. Invest Ophthalmol Vis Sci. 2000;41:417-421. 